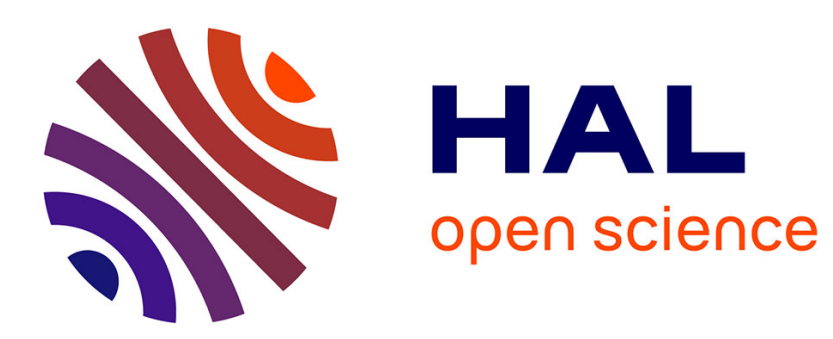

\title{
La revendication d'une paternité. Les hauts fonctionnaires français et le "style" administratif de la Commission européenne
}

Michel Mangenot

\section{- To cite this version:}

Michel Mangenot. La revendication d'une paternité. Les hauts fonctionnaires français et le "style" administratif de la Commission européenne. Pôle Sud, 2001, 15, pp.33-46. halshs-00291208

\section{HAL Id: halshs-00291208 \\ https://shs.hal.science/halshs-00291208}

Submitted on 27 Jun 2008

HAL is a multi-disciplinary open access archive for the deposit and dissemination of scientific research documents, whether they are published or not. The documents may come from teaching and research institutions in France or abroad, or from public or private research centers.
L'archive ouverte pluridisciplinaire HAL, est destinée au dépôt et à la diffusion de documents scientifiques de niveau recherche, publiés ou non, émanant des établissements d'enseignement et de recherche français ou étrangers, des laboratoires publics ou privés. 


\title{
Persée
}

http://www.persee.fr

\section{La revendication d'une paternité: Les hauts fonctionnaires français et le "style" administratif de la Commission européenne (1958-1988)}

\author{
Michel Mangenot \\ Pôle Sud, Année 2001, Volume 15, Numéro 1 \\ p. $33-46$
}

Voir l'article en ligne

Loin d'être apparu au cours des années 1980, le "style" français de la Commission européenne est le résultat d'un investissement précoce de hauts fonctionnaires français à Bruxelles. Arrivés dès 1958 dans une conjoncture très incertaine et/ou marginalisés dans leur pays, ces fonctionnaires ont fortement influencé l'administration communautaire et ses pratiques, en particulier dans son rapport au politique. Alors que cet investissement a longtemps été contrarié par les responsables de la politique européenne gaulliste, il a permis à une génération plus légitime, emmenée par Jacques Delors, de revendiquer le style administratif de la Commission. Si les positions institutionnelles et idéologiques sont marquées par une grande stabilité, c'est l'élévation du profil social et professionnel de ces hauts fonctionnaires détachés qui explique le "changement" des années 1985-1990, renouvelant ainsi l'hypothèse de path dependence.

\section{Avertissement}

L'éditeur du site «PERSEE » - le Ministère de la jeunesse, de l'éducation nationale et de la recherche, Direction de l'enseignement supérieur, Sous-direction des bibliothèques et de la documentation - détient la propriété intellectuelle et les droits d'exploitation. A ce titre il est titulaire des droits d'auteur et du droit sui generis du producteur de bases de données sur ce site conformément à la loi n`98-536 du 1 er juillet 1998 relative aux bases de données.

Les oeuvres reproduites sur le site «PERSEE » sont protégées par les dispositions générales du Code de la propriété intellectuelle.

Droits et devoirs des utilisateurs

Pour un usage strictement privé, la simple reproduction du contenu de ce site est libre.

Pour un usage scientifique ou pédagogique, à des fins de recherches, d'enseignement ou de communication excluant toute exploitation commerciale, la reproduction et la communication au public du contenu de ce site sont autorisées, sous réserve que celles-ci servent d'illustration, ne soient pas substantielles et ne soient pas expressément limitées (plans ou photographies). La mention Le Ministère de la jeunesse, de l'éducation nationale et de la recherche, Direction de l'enseignement supérieur, Sous-direction des bibliothèques et de la documentation sur chaque reproduction tirée du site est obligatoire ainsi que le nom de la revue et- lorsqu'ils sont indiqués - le nom de l'auteur et la référence du document reproduit.

Toute autre reproduction ou communication au public, intégrale ou substantielle du contenu de ce site, par quelque procédé que ce soit, de l'éditeur original de l'oeuvre, de l'auteur et de ses ayants droit.

La reproduction et l'exploitation des photographies et des plans, y compris à des fins commerciales, doivent être autorisés par l'éditeur du site, Le Ministère de la jeunesse, de l'éducation nationale et de la recherche, Direction de l'enseignement supérieur, Sous-direction des bibliothèques et de la documentation (voir http://www.sup.adc.education.fr/bib/ ). La source et les crédits devront toujours être mentionnés. 


\title{
La revendication d'une paternité \\ Les hauts fonctionnaires français et le "style" administratif de la Commission européenne (1958-1988)
}

\author{
Michel Mangenot \\ IEP de Strasbourg
}

Résumé/Abstract

Loin d'être apparu au cours des années 1980, le "style" français de la Commission européenne est le résultat d'un investissement précoce de hauts fonctionnaires français à Bruxelles. Arrivés dès 1958 dans une conjoncture très incertaine et/ou marginalisés dans leur pays, ces fonctionnaires ont fortement influencé l'administration communautaire et ses pratiques, en particulier dans son rapport au politique. Alors que cet investissement a longtemps été contrarié par les responsables de la politique européenne gaulliste, il a permis à une génération plus légitime, emmenée par Jacques Delors, de revendiquer le style administratif de la Commission. Si les positions institutionnelles et idéologiques sont marquées par une grande stabilité, c'est l'élévation du profil social et professionnel de ces hauts fonctionnaires détachés qui explique le "changement" des années 1985-1990, renouvelant ainsi l'hypothèse de path dependence.

Far from suddenly appearing in the 1980s, the French "style" of the European Commission is in fact the result of early investments made by French national civil servants when seconded to Brussels. Arriving in 1958 at a time of great incertitude and when they were marginalized within their own country, these civil servants strongly influenced the community-level administration, its practices in general and its relationship to politics in particular. If this investment was for many years resisted by members of Galullist governments responsible for European policy, it laid the groundwork for a more legitimate generation of civl servants, led by Jacques Delors, to push for this style of administration within the Commission. If the institutional and ideological positions observed are marked by great stability, it is the rise in social and professional profiles of seconded officials which provides explanations for the change observed in the 1985-90 period, a change which also necessitates the adaptation of the concept of path dependency.

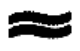

\section{Mots-Clés/Keywords}

Commission européenne, hauts fonctionnaires, path dependence, style administratif français Civil servants, European Commission, French administrative style, path dependence 
Les interrogations sur la nature de la Commission européenne ont toujours été nombreuses dans les études sur la construction européenne. Si l'intérêt pour cette institution (et son ancêtre, la Haute Autorité de la CECA), a souvent porté sur sa fonction supranationale, l'attention plus récente à sa structure, (trop) souvent présentée comme sui generis, a depuis déplacé le regard en s'intéressant à l'entité organisationnelle (Cini, 1996). Parallèlement, il s'est agi de réfléchir à la place des différentes fonctions publiques nationales au sein même du système communautaire (Scheinman, Feld, 1972; Willis, 1982; Christoph, 1993). Dans ce cadre, une question va retenir ici notre attention: le "style" administratif dit ou réputé français de la Commission européenne ${ }^{1}$. Par "style", préféré ici à "modèle" (Muller, 1992), nous n'entendons pas tant un mode de fabrication des politiques publiques (Richardson, 1982; Feick, 1990) que l'expression d'une culture administrative entendue comme l'ensemble des images de soi et des visions du monde des hauts fonctionnaires: il s'agit ainsi de la conception que ceux-ci se font de leur rôle, de leur carrière ainsi que des rapports qu'ils entretiennent avec le (et la) politique.
Si Hartmut Kaelble reconnaissait il y a quelques années "une remarquable symbiose entre les élites étatiques françaises et la bureaucratie europénne" (Kaelble, 1995, p. 130), force est de constater que ce "style" français de l'administration communautaire était en fait largement improbable. L'idée même d'une administration européenne autonome a été en effet rejetée par les responsables de la politique européenne gaulliste au cours des années soixante. C'est le modèle germanique qui a joué sur la structure initiale de la Commission, par l'action de son premier président Walter Hallstein qui s'est inspiré de la structure administrative qu'il avait lui-même établie pour l'Auswärtiges $A m t^{2}$. Hallstein, fils d'un fonctionnaire prussien et professeur de droit international, avant de rejoindre le (nouveau) ministère des Affaires étrangères à Bonn comme secrétaire d'État, a été accompagné dans ce mouvement, à Bruxelles en 1958, par Hans von der Groeben, second Commissaire allemand (en charge de la Concurrence), issu, lui, du monde de l'apprentissage mais devenu haut fonctionnaire au ministère fédéral de l'Économie, ministère qui a été cité parfois comme modèle de la nouvelle Commission de la 
CEE (Dondelinger, 1985) ${ }^{3}$. C'est ainsi que Jacques Ziller pouvait avancer récemment que "tant la structure organisationnelle de la Commission que son système de fonction publique paraissent plus proches du système allemand ou du civil service britannique" (Ziller, 2000, p. 359).

Pourtant s'est bien développée, et dès les années 1960 , une influence administrative française à la Commission européenne. Ainsi en 1973, le correspondant du Figaro d̀ Bruxelles, Yann de l'Ecotais, identifiait la mécanique communautaire comme le "fruit d'une organisation à l'allemande et d'une administration à la française" (Le Figaro, 7 juin 1973). Ce style deviendra particulièrement visible dans le milieu des années 1980 avec la relance de l'Acte unique et l'arrivée à la tête de la Commission, lors de cette seconde période charnière, de Jacques Delors (Grant, 1994), bien que Delors, tout comme Jean Monnet auparavant, ne fussent pas issus des grandes écoles prestigieuses ou des grands corps (Kaelble, 1995, p. 129). Mais pour comprendre et analyser ce style français, et plus exactement la place des hauts fonctionnaires français dans sa construction, il nous faudra revenir d la genèse de l'administration européenne, entendue ici par la mise en place de la nouvelle Commission de la Communauté économique européenne en 1958 à Bruxelles4. Les conséquences de ce lancement institutionnel apparaissent en effet décisives. Cette situation va ensuite être marquée par une grande stabilité, stabilité des positions tant institutionnelles qu'idéologiques. Ce schéma présente de ce point de vue beaucoup de similitudes avec le concept de path dependence. Ce concept, que l'on peut traduire littéralement par "dépendance au sentier" ou "sentier de dépendance", isolant quatre mécanismes conduisant à un auto- renforcement, insiste en effet sur la stabilité des processus institutionnels (Pierson, 1997) '. Le changement des années 19851990 peut alors s'expliquer par la modification du profil des acteurs qui vont s'y engager: à des "marginaux" succède en effet une nouvelle génération plus "légitime". Elle pourra alors revendiquer, plus de vingt ans après, la paternité du style administratif de la Commission. L'attention portée à l'évolution du profil social et professionnel des acteurs engagés à Bruxelles permettra ainsi d'enrichir la notion de path dependence.

\section{Les effets structurants d'une genèse incertaine}

Contrairement à une idée encore largement répandue, il y a bien eu, dès 1958, un réel investissement des hauts fonctionnaires français dans la mise en place de la nouvelle Commission de la CEE. Cet investissement a produit des effets structurants sur l'administration de la Commission et ses pratiques. On retrouve ainsi une des caractéristiques principales des phénomènes de path dependence, à savoir que les "choix initiaux" ont des effets à long terme sur les institutions. Mais la particularité est ici que le rôle des hauts fonctionnaires français n'est pas, à ce moment-là, le résultat d'une volonté stratégique: l'effet de leur présence va jouer de façon imprévue dans le cadre d'une configuration très incertaine marquée par la crise algérienne, la fin de la IV République et une conjoncture économique et financière difficile. C'est dans ce contexte qu'un groupe de hauts fonctionnaires est associé à la naissance des institutions européennes, parfois dès la négociation du Traité de Rome. Cette association n'est pas le fruit d'une volonté explicite: on dispose maintenant de nombreux 


\section{LA COMMISSION EUROPEENNE EN POLITIQUE(S)}

témoignages concordants. Ainsi Alain Prate, jeune inspecteur des finances, est envoyé à Bruxelles suite à la remarque d'un conseiller du ministre des Finances: "Il y a à Bruxelles quelques irresponsables qui prétendent instaurer une union douanière en Europe. $\mathrm{Ce}$ sont des ignorants qui ne savent pas que la situation alarmante de nos réserves en devises va nous obliger prochainement à contingenter toutes nos importations. Ces initiatives n'aboutiront à rien. Cependant, le Premier ministre, M. Guy Mollet, attache de l'importance à ces travaux, et il faut donc que quelqu'un des Finances aille voir ce qui se passe" (Prate, 1995, p. 17). La marge de manœuvre de ces jeunes hauts fonctionnaires en est alors d'autant plus importante. Un autre membre de l'équipe, Jean-Maxime Lévêque, lui aussi inspecteur des finances, évoque, lui, des "négociations étonnantes, auxquelles le gouvernement portait fort peu d'attention, accaparé qu'il était par les péripéties de la politique intérieure française et, déjà, par l'aggravation de la situation en Algérie", ainsi que les quelques hauts fonctionnaires, dont il se présente comme le benjamin, se rendant plusieurs jours par semaine à Bruxelles pour retrouver leurs collègues européens, "le plus souvent sans autres instructions que celles qu'ils se donnaient eux-mêmes" (Lévêque, 1986, p. 59).

Cette logique d'association va se rejouer dans les premiers recrutements qui s'effectuent par l'intermédiaire d'un homme, proche mais extérieur à la haute fonction publique française puisque professeur d'économie politique, sorte d'associé-rival ou de marginal-sécant (Jamous, 1968) : Robert Marjolin, nommé vice-président de la Commission en 1958, chargé des affaires économiques. Marjolin, fort de son réseau de relations européennes - il avait été nommé dix ans plus tôt à la tête de l'Organisation euro- péenne de coopération économique, première institution européenne - va jouer le rôle de recruteur. Agrégé d'économie politique, militant politique, membre depuis 1929 des Jeunesses socialistes, c'est de façon inattendue qu'il devient directeur des relations économiques extérieures en 1945. Il ne restera que très peu de temps dans la haute administration française, pour laquelle il reconnaît qu'il apparaissait comme un "intrus" (Marjolin, 1986, p. 136), mais se liera avec de nombreux hauts fonctionnaires. En 1956, il est chargé des questions européennes au cabinet du ministre socialiste des Affaires étrangères, Christian Pineau. C'est alors qu'il appelle Alain Prate: "En février 1958, le Traité de Rome était entré en vigueur; Robert Marjolin me convoqua dans le bureau qu'il occupait au Quai d'Orsay avant de rejoindre Bruxelles, où il avait été nommé vice-président de la Commission (...) 'Accepteriez-vous de venir à Bruxelles pour constituer le Comité monétaire et en assurer le secrétariat?' (...) J'acceptai donc une mission de quelques mois, qui devait en fait me retenir une dizaine d'années à Bruxelles, pendant presque toute la période de transition" (Prate, 1995, p. 40). Marjolin fera de même pour Jean-François Deniau, qu'il convainc de rejoindre Bruxelles.

La logique du recrutement est donc toujours externe. Ainsi François-Xavier Ortoli, lui aussi inspecteur des finances, alors chef de service à la Direction des relations économiques extérieures, est, lui, appelé dans un premier temps par l'autre membre de la Commission, Robert Lemaignen, pour y diriger son cabinet. "Ayant apprécié, au cours des négociations du Traité, les qualités exceptionnelles d'un jeune fonctionnaire du ministère de l'Économie, François-Xavier Ortoli, je parvins à le décider à venir à Bruxelles, du moins pour une période de 
démarrage", résume le commissaire dans ses souvenirs "de technocrate" qu'il rédige peu après son départ de Bruxelles (Lemaignen, 1964, p. 49). Membre du CNPF, Robert Lemaignen ne voulait pas en effet d'un homme du patronat mais souhaitait prendre un collaborateur "ayant la carrière, les titres et les aptitudes supposées d'un haut fonctionnaire".

Au sein de la Commission, le nouveau chef de cabinet conquiert vite un rôle singulier. Très actif, habitué aux rythmes de travail des cabinets parisiens, Ortoli y lance le modèle français de cabinet ministériel (Ritchie, 1992). L'entourage des commissaires est alors en question: d'un côté le président de la Commission, Walter Hallstein, partisan de cabinets restreints, de l'autre les commissaires français, désireux d'équipes plus étoffées. Robert Lemaignen se souvient dès 1964 que "le président se montrait par principe opposé aux cabinets nombreux: il ne voulait pas, disait-il, risquer de voir les commissaires 'médiatisés'par leurs collaborateurs immédiats. Il considérait aussi, sans doute, que tous ayant été tacitement d'accord pour que les membres des cabinets soient de la nationalité du commissaire dont ils seraient les auxiliaires, leur excessive multiplication risquerait de créer dans les cabinets un nationalisme interne, alors que son souci principal était d'assurer la collégialité des décisions et le renforcement de l'esprit communautaire qui les inspirerait" poursuit-il. (Lemaignen, 1964, pp. 49-50.)

Suite à son départ prévu du cabinet de Robert Lemaingen, une fois celui-ci lancé, la logique du hasard joue une seconde fois. Ortoli est en effet mis devant le fait accompli puisqu'au moment de ses adieux, il est nommé directeur général sans même en être averti, Marjolin ayant impérativement dû donner un nom lors d'une réunion du
Conseil des ministres. Mais les propositions de Marjolin sont parfois acceptées avec plus de difficultés: devant le peu d'enthousiasme des membres d'un service qu'il avait sollicité (le Service d'études économiques et financières du ministère des Finances), le recrutement s'est fait par tirage au sort à Paris (à la courte paille!).

À la tête de la direction du Marché intérieur, Ortoli va conquérir progressivement une certaine estime, devenant une sorte de président des Directeurs généraux. Le président Hallstein lui demande par exemple un rapport sur la manière dont la Commission a mis en place son administration. Preuve encore que la volonté de lancer une administration sur le modèle français n'est pas présente, Ortoli plaide alors, un an avant de quitter Bruxelles, pour une administration légère, très mobile, sans statut, composée de contractuels; il propose un plafonnement des services et suggère de revoir, au terme d'une première période, le recrutement effectué. Son départ pour le cabinet de Georges Pompidou, peu de temps après, l'empêchera d'être entendu. Des pans entiers de l'administration européenne resteront en effet surdimensionnés, comme la direction générale des Transports: ses membres, parmi lesquels on ne compte que quelques rares hauts fonctionnaires français, étaient, pour reprendre l'expression d'un de ces hauts fonctionnaires de l'époque, comme "dans le désert des Tartares: attendant un ennemi qui ne venait jamais, une fonction que ne venait jamais". Ainsi pour Alain Prate: "Dès le début, sous l'influence de son président, Walter Hallstein, la Commission voulut se doter d'une organisation de type étatique, en avance sur les besoins du moment. La Commission devait garder longtemps la marque originelle d'une administration quelque peu surdimensionnée, anticipant 


\section{LA COMMISSION EUROPÉNNE EN POLITIQUE(S)}

les compétences qu'elle aurait à exercer" (Prate, 1995, p. 40). En effet, dès 1958, avaient été créées, suivant la tradition administrative germanique, neuf directions générales (chacune disposant de trois directions, elles-mêmes divisées en trois divisions) : DG I : Relations extérieures; DG II : Affaires économiques et financières; DG III : Marché intérieur; DG IV: Concurrence; DG V: Affaires sociales; DG VI: Agriculture; DG VII : Transports ; DG VIII : Pays et territoires d'outre-mer; DG IX : Administration (dont une direction du budget).

Dans ce cadre, les premiers hauts fonctionnaires contribuent, malgré eux, à tracer une véritable carrière "à la française" au sein de l'administration européenne. Deniau devient vite directeur général (des négociations d'adhésion). Le poste de directeur général du Marché intérieur, chargé de mettre en place l'Union douanière, semble ensuite "réservé" aux (hauts fonctionnaires) français puisqu'il revient, au départ d'Ortoli en 1961, à Pierre Millet, administrateur civil au ministère des Finances, puis à Alain Prate et enfin à Robert Toulemon, lui aussi inspecteur des finances, entré à la Commission en 1962 par la voie du cabinet, celui de Robert Marjolin. Ce dernier tente alors, avec difficulté, de transformer la direction qui devient direction générale des Affaires industrielles ${ }^{6}$. Mais là encore ces promotions administratives ne sont pas toujours intentionnelles: la prolongation de carrière d'Alain Prate s'explique par exemple par défaut, aucun poste ne lui ayant été proposé à Paris.

En dehors du Marché intérieur ou du Comité monétaire (dépendant de la DG II, puis de la direction monétaire, créée en 1967), d'autres "sites" institutionnels sont occupés dans les mêmes conditions, comme le service juridique confié dès 1958 à Michel Gaudet, membre du Conseil d'État alors appelé par Jean Rey', le Budget (à partir de 1967), la direction générale de l'Agriculture, occupée dès 1958 par un haut fonctionnaire français (et qui le restera sans discontinuité jusqu'en 1999) et, dans un cadre différent l'Office statistique, dont la direction revient dès sa création à un fonctionnaire de l'INSEE, Raymond Dumas.

\section{La réussite d'un investisse- ment contrarié}

Comment alors expliquer la réussite de cet investissement de hauts fonctionnaires français associés de façon si improvisée à la naissance de l'administration de la CEE ? On ne peut la comprendre que par l'étude du profil social et professionnel des personnels ayant rejoint la Commission. Les quelques inspecteurs des finances ayant accepté de quitter Paris peuvent être considérés comme des "prétendants". Souvent atypiques ou en décalage par rapport au milieu de la haute bourgeoisie parisienne, Bruxelles leur a permis bien souvent de compenser un handicap de carrière. Si ce "style" français ainsi lancé a pris forme à la Commission, il va être renforcé ensuite par tout un groupe de hauts fonctionnaires "aux marges", qu'on a pu qualifier de "seconds" et qui se sont fortement investis après une période de flou pionnier permettant d'éviter le marquage et facilitant un jeu avant-gardiste. Il en va ainsi d'Émile Noël, nommé secrétaire général de la Commission, surnommé par un fonctionnaire communautaire "la Callas, l'irremplaçable, l'unique, le grand commis que la France avait prêté à l'Europe" (Buzzonetti, 1994, p. 269). Noël est entré dans l'appareil d'État par la voie extérieure: normalien (section sciences), il 
intègre le cabinet de Guy Mollet à Matignon en 1956, après avoir été chef du cabinet de ce dernier au Conseil de l'Europe à Strasbourg. C'est à ce moment seulement qu'il se met en relation avec le Secrétariat général du gouvernement, principal service administratif du Premier Ministre qui a été parfois cité comme le modèle du secrétariat (exécutif puis) général ${ }^{8}$ de la Commission, à la tête duquel il resta jusqu'en 1986 (Cassese, 1987, pp. 12-13). Le premier chef de cabinet de Robert Marjolin, vice-président, Jean-Claude Richard, est un jeune secrétaire des Affaires étrangères, peu aguerri, ancien enseignant entré à l'ENA par concours interne. En 1962, il est remplacé par Jean Flory, fraîchement diplômé de l'Institut d'études politiques de Paris.

Le cas de Georges Rabot, directeur général de l'Agriculture de 1958 à 1978, est similaire. Haut fonctionnaire du ministère de l'Agriculture (il y resta sous-directeur), il n'est pas membre du corps du GREF mais agronome. Ayant, selon les propres termes d'un de ses collègues français à Bruxelles, "souffert de l'administration française" (entretien), il était, avant son départ pour la Commission, directeur à l'Organisation européenne de coopération économique (OECE). Si l'on considère que la $\mathrm{PAC}$ allait devenir, à partir de 1960, la seule priorité de la politique européenne gaulliste, l'investissement de Rabot dans les questions agricoles européennes apparaît comme largement antérieur à "l'intérêt" de de Gaulle. S'identifiant à sa fonction, atteint par la limite d'âge, il quitte son poste pour décéder trois semaines plus tard (Le Monde, 23-24 juillet 1978). À la direction du Budget est nommé en 1973 Daniel Strasser, ancien chargé de mission dans les services du Premier ministre, entré à la Commission en 1958 comme administrateur principal. On peut citer aussi le cas
d'Armand Saclé à la direction générale de la Concurrence: directeur des aides d'État pendant dix ans, issu d'un "petit concours" du contrôle économique pendant la guerre, il est aussi "second" dans le sens où il a été désigné suite au refus d'un membre, plus titré, des Finances initialement pressenti par Marjolin. Au service juridique, il faut noter le parcours de Gérard Olivier, sous-préfet, qui en 1970 succède (comme directeur général adjoint) à Michel Gaudet, celui-ci ayant décidé de quitter la Commission une fois l'adhésion britannique acquise.

Les membres de cette génération ont pu faire de véritables carrières européennes grâce aux ressources retirées du label générique d'énarques ou de membres de certains départements ministériels prestigieux comme le ministère des Finances, les hiérarchies internes "nationales" disparaissant en quelque sorte à Bruxelles. Ils ont pu alors consolider leurs positions grâce en particulier à un haut niveau de rémunérations: les différences importantes de traitements entre les deux administrations sont en effet restées longtemps ignorées à Paris, inquiétant seulement Michel Debré qui est intervenu vigoureusement sur ce "danger" au Conseil d'administration de l'ENA en 1958. Demeurés attachés à la France - beaucoup auraient préféré y faire carrière et la plupart y sont rentrés la terminer après leur départ de la Commission - ces hauts fonctionnaires ont contribué ainsi à légitimer un certain modèle français à Bruxelles, ayant la volonté de faire bonne figure par rapport à leurs collègues restés à Paris et qui les voyaient souvent d'un mauvais œeil.

Car le paradoxe est que cet investissement est alors contrarié par les responsables de la politique européenne de l'époque. La contribution officielle française à l'élaboration du statut type de la fonction publique euro- 


\section{LA COMMISSION EUROPEENNE EN POLITIQUE(S)}

péenne, au début des années 1960 , est, de ce point de vue, très significative'. Les représentants des ministères des Affaires étrangères et des Finances refusèrent par exemple de participer à la première réunion de la Conférence gouvernementale sur la Fonction publique européenne en novembre 1963, à Luxembourg. La justification était double: la présence de représentants des Communautés européennes en qualité de membres de droit, au même rang que les gouvernements, ainsi que le caractère supranational (votes majoritaires) qui s'attacherait aux recommandations formulées par la Conférence. Le danger redouté est explicitement celui de "l'apparition d'une véritable carrière dans les organisations européennes ${ }^{10}$ ". Même plus tard, au moment de la fusion des exécutifs, en 1967, la volonté du représentant permanent français, Jean-Marc Boegner, sera de "demander que l'on revienne sur la conception fâcheuse de la carrière européenne "1". L'administration française chercha même à "faire payer" leur carrière à la Commission aux fonctionnaires français désireux de rentrer ${ }^{12}$, et certains préférèrent alors rejoindre le privé. Seule la presse souligna l'intérêt d'une forte influence française sur (et dans) l'administration de la Commission : en 1968, un envoyé spécial du Monde à Bruxelles termina sa grande enquête en trois volets sur les "Communautés européennes à l'heure de la fusion" par un article intitulé "Un atout maître: le français" qui se termine par ces mots: "La Commission et ses services offrent à la France un instrument privilégié pour essayer de faire adopter sa manière de voir, d'imposer subtilement sa manière d'être. Pour un pays comme la France qui est encore mal armé pour la compétition internationale, une place de premier choix aux commandes de la machine européenne fournit le moyen de compenser sur l'aire des
Six sa relative déficience économique. À cet égard, si le 'machin'communautaire n'existait pas, c'est la France qui aurait le plus d'intérêt à l'inventer" (Le Monde, 19/01/1968).

$\mathrm{Au}$ total, ces investissements ont néanmoins rendu "naturel" le cadre de la Commission européenne pour les hauts fonctionnaires français. Il ne s'agit plus de lutter contre cette forme institutionnelle de l'Europe qu'est la Commission - tentative présente chez de nombreux hauts fonctionnaires de la période gaulliste -, mais d'y convoiter des débouchés professionnels. Ce qui va être le cas en 1973 justement, quand François-Xavier Ortoli en devient le président, entouré d'un large éventail de membres des grands corps formant son cabinet. Si le journal Combat parle alors de "la fin des Français à Bruxelles" (Combat, 26 février 1973), c'est pour stigmatiser le départ de la première génération de fonctionnaires français. Coïncidant avec l'arrivée des fonctionnaires britanniques, ce moment a joué comme premier révélateur du style français de la Commission. Ainsi le poste de directeur général de l'administration et du personnel est alors revendiqué, et revient pour la première fois à un haut fonctionnaire français, Pierre Baichère, administrateur civil du Trésor entré dans l'administration européenne en 1959 mais à la Haute Autorité à Luxembourg, et devenu en 1968 directeur du budget dans la Commission unifiée $^{13}$. En 1981, le poste revient à un autre fonctionnaire français (INSEE) de la première heure à Bruxelles, Jean-Claude Morel, qui était depuis 1958 à la direction générale des Affaires économiques et financières et dont il venait d'être nommé directeur adjoint. Il a alors la charge de mettre en application le rapport Spierenburg sur la réforme de l'administration de la Commission. 
Avec la promotion d'Ortoli comme président (il sera vice-président chargé des affaires économiques et financières de 1977 à 1985), précédée de celle de Jean-François Deniau comme commissaire en 1967 - tous deux anciens inspecteurs des finances et anciens hauts fonctionnaires de la Commission - et accompagnée en 1973 de celle de Claude Cheysson, membre du Quai d'Orsay, c'est aussi la formalisation d'un processus de définition du poste de Commissaire qui se réalise. Jusqu'à de Silguy (diplomate et ancien du cabinet d'Ortoli) en 1995, et Lamy en 2000, le profil du commissaire "à la française", en opposition à la très grande majorité des autres cas ${ }^{14}$, est avant tout celui d'un haut fonctionnaire ${ }^{15}$, proche du politique mais sans véritable onction électorale ${ }^{16}$. La volonté "politique", voulant ici cantonner la Commission dans un rôle "technique" - Pompidou expliquait le choix d'Ortoli en mettant en avant son expertise économique -, vient renforcer le poids des hauts fonctionnaires français à Bruxelles.

Si cet investissement de hauts fonctionnaires a "réussi", c'est aussi en raison de la relative faiblesse structurelle des hautes fonctions publiques des pays membres, avant l'adhésion britannique. En dehors du cas allemand déjà évoqué, mais malgré tout largement en voie de reconstitution à Bonn et freiné par l'absence jusqu'aux années 1970 de rôle réel de la DG IV, les hautes administrations européennes, l'Italienne, qualifiée parfois "d'élite modeste" (Righettini, 1995), et la néerlandaise, souffraient d'une grande hétérogénéité et d'une absence de prestige social. Dans le second cas s'ajoutait l'obligation, instaurée dès la CECA, de démissionner de l'administration néerlandaise ${ }^{17}$. Parmi leurs homologues, les hauts fonctionnaires français apparaissent de plus les plus généralistes par rapport à des profils exclusivement juristes (comme à la Concurrence où Saclé peut jouer de sa compétence économique) ou économistes, d'autant que leurs postes sont souvent transversaux. Enfin, cette réussite s'explique aussi en raison de l'adéquation de leur profil idéologique. Les premiers hauts fonctionnaires français affectés à Bruxelles sont en effet parmi les plus libéraux, opposés en particulier au modèle économique de la planification qui se met en place en France. Ils se définissent en opposition aux hauts fonctionnaires "planificateurs", ralliés à la nouvelle Cinquième République (Gaîti, 1998; Dulong, 1998) et accusés d'un excessif "dirigisme" ${ }^{18}$. Alain Prate explique ainsi la réponse positive qu'il donne à Marjolin, quand ce dernier lui demande de partir pour Bruxelles, en pointant les modes de pensée qui "étaient entièrement différents de ceux qui prévalaient 'alors'dans l'administration française, particulièrement au ministère des Finances: dans les années cinquante, les responsables de l'Économie en France étaient uniformément orientés vers la planification et la gestion, par des mesures d'inspiration dirigiste, d'une économie strictement fermée aux échanges, aussi bien pour les marchandises que pour les capitaux". "La notion d'économie de marché ouverte sur l'extérieur, et dont l'équilibre soit assuré par une action globale sur les finances publiques et sur la monnaie, leur était étrangère" ajoute-t-il (Prate, 1995, pp. 40-41) ${ }^{19}$. Si ces remarques accréditent l'existence d'un tournant libéral (européen) avant le "tournant néo-libéral" des années 1980 , elles nous informent sur une autre dimension de la marginalité des premiers hauts fonctionnaires français. D'orientation libérale, ceux-ci se sont sentis "déphasés" en France où triomphent alors les idées keynésiennes et où les "modernisateurs" tentent d'enrôler de Gaulle dans 
une nouvelle défense de la planification. Mais cette marginalité à Paris est un atout à la Commission où règne une certaine aversion pour toute forme de dirigisme économique. L'identité souvent relevée entre l'esprit modernisateur et interventionniste de l'administration française et les Communautés européennes (Kaelble, 1995, p. 130) apparaît largement infondée ${ }^{20}$. Les positions idéologiques des hauts fonctionnaires français engagés dans la période dite de relance, avec l'Acte Unique, resteront sur la même ligne. Mais le référentiel "de marché" est désormais largement partagé à Paris (Jobert, 1994).

\section{Stabilité des positions, évo- lution des profils}

Ce qui frappe alors quand on compare les positions des hauts fonctionnaires français détachés à la Commission européenne, entre les débuts (1958-1960) et les années 1986-1988, c'est une grande stabilité. Stabilité dans le nombre tout d'abord: une soixantaine en 1958, puis une quarantaine en 1985 et une cinquantaine en 1990 (les chiffres augmentent ensuite si l'on prend en compte les Experts nationaux détachés, ces nouveaux postes de détachement lancés en 1988). Stabilité aussi dans les positions institutionnelles: les postes occupés sont à peu de choses près les mêmes. Si aucune des positions tant institutionnelles qu'idéologiques des hauts fonctionnaires français au sein de la Commission européenne n'apparaissent changées - c'est le système de référence national qui a varié -, la modification des profils des acteurs est, elle, effective. Cela vient ici renforcer en même temps que renouveler l'approche en termes de path dependence, puisqu'il semble que ce soient les seuls investissements des premiers hauts fonctionnaires français à Bruxelles, en marges - investissements par ailleurs longtemps contrariés - qui aient créé des "sentiers de dépendance".

Le même phénomène de personnalité marginale-sécante se rejoue cette fois-ci, pour le recrutement, avec Jacques Delors. Nommé président en 1985, tout en se réservant les affaires monétaires, celui-ci apparaît dans la même position que, trente ans avant lui, Robert Marjolin : politiquement engagé (au Parti socialiste), sans être haut fonctionnaire, il a eu une carrière "experte" au Plan. Mais la différence est ici la démarche volontaire de son équipe, dont le profil social et professionnel est surtout plus élevé. Au niveau du cabinet tout d'abord. Delors appelle Pascal Lamy, inspecteur des finances, énarque donc mais aussi diplômé de HEC, membre de la direction la plus prestigieuse du ministère des Finances, celle du Trésor, et sortant du cabinet du Premier Ministre. Il sera surnommé I'"Exocet ${ }^{21 "}$. Il sera accompagné de Jérôme Vignon, polytechnicien, sortant directement du cabinet de la rue de Rivoli (avant que celui-ci ne prenne la direction de la Cellule de prospective en 1989) puis à partir de 1991 d'un autre inspecteur des finances, Jean-Pierre Jouyet quittant alors le cabinet de Roger Fauroux. Les transformations de profil des directeurs de l'Agriculture sont elles aussi significatives. À Louis Rabot, haut fonctionnaire "technique", succède en 1978 Claude Villain, directeur général de la Concurrence au ministère de l'Économie mais ancien de la France coloniale, puis en 1984 Guy Legras, diplomate, chef du service de la Coopération économique au ministère des Affaires étrangères (responsable de la politique européenne) ${ }^{22}$. En 1987, au moment du départ d'Émile Noël du secrétariat général, Jean-Louis 
Dewost, conseiller d'État, est nommé directeur général du Service juridique. La même année Yves Franchet, inspecteur général de l'INSEE, prend la tête de l'Office statistique, devenu Eurostat. Polytechnicien (à vingt et un ans), celui-ci est un ancien directeur de l'École nationale de la statistique et de l'administration économique. En 1989, un administrateur civil du Trésor, Jean-Paul Mingasson, succède à Daniel Strasser comme directeur général des Budgets. Le poste que ce dernier occupait - directeur des Affaires monétaires revient à un autre administrateur civil du Trésor, Jean-François Pons, à la fois ancien de l'ENA et d'HEC, qui deviendra en 1994 directeur adjoint de la Concurrence.

Cette nouvelle génération, plus légitime, peut trouver alors, se réapproprier en fait, une forte empreinte française dans l'administration européenne. Pascal Lamy explique par exemple que l'adaptation de la haute fonction publique française en a été facilitée "parce que la Commission a été fabriquée par des hauts fonctionnaires français, ce qui leur a donné pendant très longtemps un avantage comparatif certain sur les autres. C'est plutôt dans ce sens-là qu'est la causalité. Le produit Commission, l'organisation de ses services, ses procédures, sa langue, etc., a quand même été fabriqué sur le modèle français, alors mâtiné d'éléments pas du tout français, comme la collégialité" (entretien 25/05/98). L'élément singulier est ici que les hauts fonctionnaires qui ont donné à la Commission son style administratif sont loin d'être des représentants de l'excellence administrative française et que leur parcours a longtemps été dénié. En se réappropriant leur trajectoire, cette seconde génération peut se réaffirmer sur la scène européenne dans un contexte de concurrence accrue entre modèles administratifs. L'étude de ces investissements permet alors de mieux comprendre certains mécanismes concrets de l'institutionnalisation de la Commission.

1. Cet article, issu d'une communication au colloque

\section{Notes}

Intégrer l'Europe dans l'analyse des politiques publiques du 28 avril 2000 organisé par Pascale Laborier et Bruno Palier (CRPS-CEVIPOF), procède plus généralement de notre thèse de doctorat en science politique: (Mangenot, 2000). On ne disposait jusque-là que de quelques éléments dans de tares travaux juridiques: Dubouis (1975) et Vignes (1975).

2. Cette inspiration s'est parfois manifestée dans des détails très précis, Hallstein allant jusqu'à reproduire un système d'écriture à couleurs d'encres différentes utilisé à Bonn. (Noël, 1992, pp. 150-151).

3. Pour J. Dondelinger, alors représentant permanent du Luxembourg à Bruxelles, le "concept de l'Administration de la CEE était calqué sur le schéma d'organisation du Bundeswirtschaftsministerium", alors que les concepts d'administration de la CECA et de l'Euratom étaient des transpositions respectivement du Commissariat français au Plan et du Commissariat français de l'Énergie Atomique (p. 90).

4. On ne dispose encore, en effet, que de peu d'informations sur les débuts de la Commission européenne. À noter seulement: Cassese, Della Cananea, 1992. Le doyen Georges Vedel appelait récemment de ses vœux une analyse sociologique de la mise en place de cette nouvelle bureaucratie (Commission européenne, 1999, p. 53, "Les Communautés et leur sociologie administrative").

5. Pour une présentation en français, $c f$. Palier et Bonoli (1999, pp. 400-403), ainsi que Surel (2000, pp. 135-149).

6. En 1967, la DG III change de nom et devient la direction générale des Affaires industrielles. La même année, la DG XIV Marché intérieur et rapprochement des législations est créée elle deviendra en 1973 la DG XI. En 1977, la DG III redevient la direction générale du Marché intérieur (et des affaires industrielles).

7. Michel Gaudet avait déjà constitué le service juridique de la Haute Autorité à Luxembourg.

8. En 1958, la fonction était intitulée secrétaire exécutif. Elle prendra le nom de secrétaire général en 1967, lors de la fusion des exécutifs, et restera confiée à Emile Noël jusqu'en 1986. 
9. Déjà en 1955, lors de la rédaction du premier statur de la fonction publique de la CECA, le nouveau président de la Haute Autorité, René Mayer, ancien haut fonctionnaire français, avait retiré la disposition élaborée par Jean Monnet consistant en l'incompatibilité de la persistance d'un lien conservé avec les administrations nationales (Conrad, 1992, p. 69).

10. Note d'information sur la participation de la France à l'élaboration du statut type de la Fonction publique européenne, 28 mai 1964, Bureau des Organismes internationaux, Direction du Budget, Ministère des Finances (Archives économiques et financières, B10745).

11. Télégramme, Représentation française auprès des Communautés européennes, Bruxelles, 21 septembre 1967 (Archives économiques et financières, B10745).

12. Certains ont alors intégré les nouvelles Chambres régionales des comptes créées en 1982.

13. Daniel Strasser était assistant du directeur général de l'Administration dès 1958, puis directeur du personnel en 1968, avant de rejoindre le Budget.

14. Les rares exceptions, en dehors du cas de Hans von der Groeben (1958-1970), sont celles des Luxembourgeois Albert Borschette (1970-1976) et Jean Dondelinger (1989-1993), d'Étienne Davignon (1981-1985), du premier commissaire grec Georges Kontogeorgis (1981-1985) ou, plus récemment, de Marcelino Oreja (19952000).

15. À l'exception de Raymond Barre (1967-1973) ou d'Edith Cresson (1995-2000), les autres figures, n'étant pas issues de l'administration, ont joué un rôle moins déterminant: Robert Lemaignen (1958-1961), Henri Rochereau (1961-1970), Edgard Pisani (1981-1984) ou Christiane Scrivener (1989-1994).

16. Certains d'entre eux tentèrent pendant leur mandat le baptême des urnes mais sans succès, comme, aux élections législatives de 1962, Robert Marjolin sous une étiquette socialiste (Le Monde, 01/12/1962) ou en 1968 Jean-François Deniau sous une étiquette gaulliste.

17. Edmond Wellenstein raconte ainsi que son ministre lui demanda de choisir et qu'il dut démissionner du poste qu'il occupait au ministère néerlandais des Affaires étrangères pour intégrer la Haute Autorité de la CECA (Commission européenne, 1999, pp. 67-68).

18. On a retrouvé en Grande-Bretagne le même type de structure d'oppositions au Marché commun quand le leader du parti travailliste, Hugh Gaitskell, dans un célèbre discours, expliqua que l'adhésion à la CEE empêcherait l'objectif principal du Labour : l'établissement d'une planification rigoureuse. On peut aussi interpréter le refus de la CECA par le Royaume-Uni au motif que les deux secteurs considérés, le charbon et l'acier, étaient nationalisés.

19. Alain Prate termine en stigmatisant l'enseignement de l'ENA (sur ce débat: Mangenot, 2001) et en revalorisant la figure de Jacques Rueff qui était selon lui "traité avec condescendance par le monde officiel". Cet inspecteur des finances, ancien directeur du Trésor avant la guerre et apôtre du libéralisme, a été lui aussi en poste dans une institution européenne, en tant que juge à la Cour européenne de justice de Luxembourg de 1952 a 1962. Les postes investis par les hauts fonctionnaires sont aussi ceux qui permettent une politique libérale. Ainsi le poste de directeur du Marché intérieur, consistant à mettre en place l'Union douanière et à négocier le tarif extérieur commun, a permis à son premier titulaire de demander à la France qu'elle supprime ses contingents d'importations, entraves aux échanges. L'exception principale est le poste de directeur de la Sécurité sociale et de l'action sociale (une des trois directions de la DGV), occupé de 1958 à 1973 par Jacques Ribas, maître des requêtes au Conseil d'État.

20. Certains d'entre eux, comme Claude Gruson, essayeront bien depuis Paris de diffuser, mais en vain, à Bruxelles, un modèle de planification "à la française". Cette impossible planification européenne donnera lieu à une déclaration de la Commission sur la programmation européenne puis à la création du Comité de politique à moyen terme. On a surtout retrouvé cette influence à la Haute Autorité de Luxembourg dans ses dernières années, quand celle-ci, sur le déclin, a été redécouverte par certains hauts fonctionnaires planificateurs (Simon Nora en est devenu en 1960 le directeur général de l'Économie) avant de laisser en 1967 un "testament dirigiste". Auparavant, l'influence institutionnelle du (Commissariat au) Plan semble avoir été vite écartée (Mazey, 1992) d'autant que le poste initialement investi était la direction financière.

21. Voir son portrait à l'occasion de sa nomination comme Commissaire: "L'Exocet retourne à Bruxelles", La Tribune, 9 juillet 1999.

22. Voir "Guy Legras, le négociateur discret de la politique agricole commune", Le Monde, 23 février 1999. 


\section{Références}

Buzzonetti M., "Merci, Monsieur Noë", in Martin S. (ed.) The Construction of Europe. Essays in Honour of Emile Noël, Dordrecht, Kluwer Academic Publishers, 1994.

Cassese S., "Divided powers: European administration and national bureaucraties", in Cassese S. (ed.), The European administration, Bruxelles, IISA-IEAP, 1987.

Cassese S., Della C., "The Commission of the European Economic Community: the Administrative Ramifications of its Political Development (1957-1967)", in Annuaire d'histoire administrative européenne, $\mathrm{n}^{\circ} 4$, Baden-Baden, Nomos Verlag, 1992.

Christoph J. B., "The effects of Britons in Brussels: The European Community and the Culture of Whitehall", Governance, Octobre 1993.

Cini M., The European Commission: leadership, organisation and culture, Manchester, Manchester University Press, 1996.

Commission européenne, Quarante ans des Traités de Rome ou la capacité des traités d'assurer les avancées de la construction européenne, Bruxelles, Etablissements Emile Bruylant, 1999.

Conrad Y., "La Communauté européenne du charbon et de l'acier et la situation de ses agents. Du régime contractuel au régime statutaire (1952-1958)", in Annuaire d'histoire administrative européenne, op. cit.

Dierickw G., Beyers J., "Belgian Civil Servants in the European Union: A Tale of Two Cultures", West European Politics, July 1999.

Dondelinger J., "Relations avec les administrations nationales", in Jamar J., Wessels W. (eds.), L'administration communautaire d l'beure du choix, Bruges, De Tempel, 1985, p. 89-98.

Dubouis L., "L'influence française sur la fonction publique européenne", in Rideau J. (ed.), La France et les Communautés européennes, LGDJ, 1975.

Dulong D., Moderniser la politique. Aux origines de la V' République, Paris, L'Harmattan, 1998.

Feick J., "L'analyse comparative des politiques publiques: un chemin vers l'intégration des résultats?", L'année sociologique, $\mathrm{n}^{\circ} 40,1990$.

Gaïti B., De Gaulle, prophète de la Cinquième République, Paris, Presses de Sciences-Po, 1998.

Grant C., Delors. Inside the House that Jacques Built, Londres, Nicholas Brealey, 1994.

Jamous $\mathrm{H}$., Contribution à une sociologie de la décision: la reforme des études médicales et des structures hospitalières, Copédith, 1968.

Jobert B. (dir.), Le tournant néo-libéral en Europe, Paris, L'Harmattan, 1994.

Kaelble H., "Á propos des divers modèles d'élites", in Théret B. (dir.), L'État, la finance et le social. Souveraineté nationale et construction européenne, Paris, La Découverte, 1995.

Lemaignen R., L'Europe au berceau... Souvenirs d'un technocrate, Paris, Plon, 1964.

Lévêque J.-M., En première ligne, Paris, Albin Michel, 1986.

Mangenot M., Une Europe improbable. Les hauts fonctionnaires français dans la construction européenne (19481992), thèse en science politique, Université Robert Schuman, Institut d'études politiques de Strasbourg, décembre 2000.

Mangenot M., " $\mathrm{La}$ formation à l'Europe des hauts fonctionnaires des Finances français entre économie mondiale et planification nationale", in Poidevin R., Girault R. (dir.)., Le rôle des ministères des Finances et des ministères de l'Économie dans la construction européenne (1957-1978), Paris, Comité pour l'histoire économique et financière de la France, 2001.

Marjolin R., Le travail d'une vie. Mémoires (1911-1986), Paris, Robert Laffont, 1986.

Mazey S., "Conception and Evolution of the High Authority's Administrative Services (1952-1956): from Supranational Principles to Multinational Practices", in Annuaire d'histoire administrative européenne, op. cit.

Muller P., "Le modèle français d'administration face à la constitution d'un espace public européen", in Muller P. (dir.), L'administration française est-elle en crise?, Paris, L'Harmattan, 1992.

Noël E., "Témoignage: l'administration de la Communauté européenne dans la rétrospective d'un ancien haut fonctionnaire", in Les débuts de l'administration de la Communauté européenne, op. cit.

Palier B., Bonoli G., "Phénomènes de path dependence et réformes des systèmes de protection sociale", Revue française de science politique, vol. $49, \mathrm{n}^{\circ} 3$, juin 1999.

Pierson P., "Increasing Returns, Path Dependence and the Study of Politics", Jean Monnet Chair Papers, $\mathrm{n}^{\circ} 44$, Centre Robert Schuman, Institut universitaire européen de Florence, 1997.

Prate A., La France en Europe, Paris, Economica, 1995. 


\section{LA COMMISSION EUROPEENNE EN POLITIQUE(s)}

Richardson J. (dir.), Policy Styles in Western Europe, Londres, Allen and Unwin, 1982.

Righettini S., "Les dirigeants de la haute fonction publique italienne. Une élite modeste?", in Suleiman E., Mendras H. (dir.), Le recrutement des élites en Europe, Paris, La Découverte, 1995.

Ritchie E., "The Model of French Ministerial Cabinets in the Early European Commission", in Annuaire d'bistoire administrative européenne, op. cit.

Scheinman L., Feld W., "The European Economic Community and National Civil Servants of the Member States", International Organization, vol. 26, $\mathrm{n}^{\circ} 1,1972$.

Stevens A., Brussels Bureaucrats? The Administration of the European Union, Londres, Palgrave, 2001.

Surel Y., "Comparer des sentiers institutionnels. Les réformes des Banques centrales au sein de l'Union européenne", Revue internationale de politique comparée, vol. 7, $\mathrm{n}^{\circ} 1,2000$.

Vignes D., "Les fonctionnaires communautaires de nationalité française", in La France et les Communautés européennes, op. cit..

Ziller J., "De la nature de l'administration européenne", Revue française d'administration publique, $\mathrm{n}^{\circ}$ 95, juilletseptembre 2000. 